TRANSACTIONS OF THE

AMERICAN MATHEMATICAL SOCIETY

Volume 360, Number 7, July 2008, Pages 3857-3873

S 0002-9947(08)04472-3

Article electronically published on February 27, 2008

\title{
WEAK UNCERTAINTY PRINCIPLE FOR FRACTALS, GRAPHS AND METRIC MEASURE SPACES
}

\author{
KASSO A. OKOUDJOU, LAURENT SALOFF-COSTE, AND ALEXANDER TEPLYAEV
}

\begin{abstract}
We develop a new approach to formulate and prove the weak uncertainty inequality, which was recently introduced by Okoudjou and Strichartz. We assume either an appropriate measure growth condition with respect to the effective resistance metric, or, in the absence of such a metric, we assume the Poincaré inequality and reverse volume doubling property. We also consider the weak uncertainty inequality in the context of Nash-type inequalities. Our results can be applied to a wide variety of metric measure spaces, including graphs, fractals and manifolds.
\end{abstract}

\section{INTRODUCTION}

The weak uncertainty inequality recently introduced in 30 for functions defined on p.c.f. fractals in general, and on the Sierpiński gasket in particular, obeys the same philosophy as the classical uncertainty principle: it is impossible for any normalized function to have a small energy and to be highly localized in space. We refer to [13, 31, 37] for more background on uncertainty principles. However, the existence of localized eigenfunctions on some of these fractals (see [10, 12, 35, 43]) is a main obstacle in proving any analogue of the classical Heisenberg inequality. In this paper we introduce a new approach to prove weak uncertainty principles for functions defined on metric measure spaces equipped with a Dirichlet (or energy) form, which include certain fractals and fractal graphs such as the Sierpiński lattice. More precisely, we show that the weak uncertainty principle holds on all spaces equipped with an effective resistance metric and a measure satisfying an appropriate growth condition. Additionally, we show that if instead of the existence of an effective resistance metric on the space, we assume that a Poincaré or a Nash inequality holds along with another appropriate growth condition on the measure, then it is also possible to prove the weak uncertainty principle in this setting. In particular, our results show that the self-similarity of the measure, which was heavily used in [30, can be replaced by weaker conditions.

In order to formulate any uncertainty inequality, one has to define measures of space and frequency concentration. For example, for complex-valued functions on

Received by the editors August 15, 2006.

2000 Mathematics Subject Classification. Primary 28A80, 42C99; Secondary 26D99.

Key words and phrases. Uncertainty principle, p.c.f. fractal, Heisenberg's inequality, measure metric spaces, Poincaré inequality, self-similar graphs, Sierpiński gasket, uniform finitely ramified graphs.

The second author was supported in part by NSF grant DMS-0603886.

The third author was supported in part by NSF grant DMS-0505622. 
$\mathbb{R}$ the classical Heisenberg Uncertainty Principle states that

$$
\operatorname{Var}\left(|\hat{f}(\xi)|^{2}\right) \operatorname{Var}\left(|f(x)|^{2}\right) \geqslant \frac{1}{16 \pi^{2}}
$$

for any function of $f \in L^{2}(\mathbb{R})$ such that $\|f\|_{2}=1$ and where $\hat{f}$ denotes the Fourier transform on $\mathbb{R}$. This inequality can be rewritten in the following form:

$$
\int_{\mathbb{R}} \int_{\mathbb{R}}|x-y|^{2}|f(x)|^{2}|f(y)|^{2} d x d y \int_{\mathbb{R}}\left|f^{\prime}(x)\right|^{2} d x \geqslant \frac{1}{8}
$$

for any function of $L^{2}$-norm one. We refer to the survey article [13] for more information on the uncertainty principle.

In this paper we consider a metric measure space $(K, d, \mu)$; that is, $(K, d)$ is a metric space equipped with a Borel measure $\mu$. If $\mathcal{E}$ is an energy form on this metric measure space, then we will say that a weak uncertainty principle holds on $K$ if the following estimate

$$
\operatorname{Var}_{\gamma}\left(|u|^{2}\right) \mathcal{E}(u, u) \geqslant C
$$

holds for any function $u \in L^{2}(K) \cap \operatorname{Dom}(\mathcal{E})$ such that $\|u\|_{L^{2}}=1$. Here $C$ is a constant independent of $u$, and the spacial variance is defined by

$$
\operatorname{Var}_{\gamma}\left(|u|^{2}\right)=\iint_{K \times K} d(x, y)^{\gamma}|u(x)|^{2}|u(y)|^{2} d \mu(x) d \mu(y) .
$$

The central question of our paper is the relation between $d, \gamma, \mu$ and $\mathcal{E}$ which implies the weak uncertainty principle, assuming that the measure $\mu$ satisfies an appropriate growth condition. We formulate sufficient conditions in several situations. The first one is when $d$ is the so-called effective resistance metric on $K$ and satisfies certain scaling properties. This setting is particularly relevant in the context of analysis on fractals associated with $\mathcal{E}$ and fractal graphs; see [4, 21, 22, 23. for more on the effective resistance metric. In this case $\gamma=b+1$, where $b$ is an exponent which often plays the role of a dimension. Our result not only provides a different and simpler proof of [30, Theorems 1 and 2], but also extends them to all p.c.f. fractals 20, 22 and fractal graphs [9, 14, 15, 17, 25, 28, 29], as well as to modifications of them such as some fractafolds [39, 41]. Additionally, our result recovers the classical Heisenberg Uncertainty Principle in $\mathbb{R}$, although not with the best constant. We also consider situations where the effective resistance metric does not exist. In these cases we assume some volume conditions and either that there is a certain scaling in Poincaré's inequality, or that a Nash-type inequality holds. Either of these conditions allows us to prove our result. These latter results are applicable for a wide variety of metric measure spaces, ranging from graphs to elliptic operators on manifolds. Note that, in this case, the number $\gamma$ appearing in (2) cannot, in general, be interpreted as a dimension in the usual sense. However, $b=\gamma-1$ will often represent the so-called walk dimension that appears frequently in recent works on heat kernel estimates (see 9 and the references therein). One of the features of our results is their robustness. For example, since the weak uncertainty principle holds for the Sierpiński graphs, it also holds for the manifolds with similar structures, e.g., the fractal-like manifolds considered in [27]. Roughly speaking, the weak uncertainty principle holds if the energy and measure have polynomial-type behavior, such as in the case of fractals, fractal graphs and groups of polynomial growth. 
Our paper is organized as follows. In Section 2 we state and prove our main results. In particular, we prove that the weak uncertainty principle holds under a variety of conditions ranging from the existence of an effective resistance metric to assuming Nash or Poincaré inequalities. Section 3 describes a few metric measure spaces for which the main results of Section 2 can be applied: p.c.f. fractals, uniform finitely ramified graphs, Sierpiński carpets, fractal-like manifolds. We also discuss the relation with recent results on the heat kernel estimates on metric measure spaces.

Among the many other generalizations of the Heisenberg uncertainty principle we wish to point out a recent preprint [11, in which the authors work in the setting of Lie groups with polynomial volume growth and which partially overlaps with our results.

\section{MAIn RESUlts}

Let $(K, d)$ be a metric space equipped with a measure $\mu$ and a positive-definite symmetric quadratic form $\mathcal{E}$ with domain $\operatorname{Dom}(\mathcal{E}) \subset L^{2}(K)$. Later, we will impose some conditions relating the distance $d$ to the form $\mathcal{E}$. We denote by $B_{r}(x)$ the ball with center $x$ and radius $r$ in the metric $d$. To simplify notation, we make the convention that the $L^{2}$-norm is infinite if a function is not square integrable, and that the energy form is infinite if a function is not in its domain.

2.1. Weak uncertainty principle and effective resistance metric. In this subsection, we assume that $\mathcal{E}$ is a Dirichlet form and that the metric measure space $(K, d, \mu)$ is such that $d$ is the effective resistance metric associated to $\mathcal{E}$. The most detailed study of such spaces, where $\mathcal{E}$ is also called a resistance form, is 23.

The effective resistance metric is defined by

$$
d(x, y)=\sup \mathcal{E}^{-1}(u, u),
$$

where the supremum is taken over all continuous functions $u$ such that $u(x)=$ $1, u(y)=0$. The existence of the effective resistance metric is a nontrivial problem; see [3, 4, 5, 6, 21, 22. In particular, it is worth noticing that there are spaces without an effective resistance metric, that is, for which the quantity above is infinite, e.g., for $\mathbb{R}^{n}$ with $n \geqslant 2$. However, on p.c.f. fractals and on some Sierpiński carpets, which are "not far from being one dimensional", it is known that the effective resistance metric does exist (see Subsection 3.4).

We now state our first main result, which generalizes Theorems 1 and 2 of 30 .

Theorem 1. Let $K$ be a space equipped with a measure $\mu$ and the effective resistance metric $d$ associated to the Dirichlet form $\mathcal{E}$. Assume that there exist positive constants $b, C_{1}, C_{2}$ such that for all $x \in K$ and $r>0$ the following inequalities hold:

$$
C_{1} r^{b} \leq \mu\left(B_{r}(x)\right) \leq C_{2} r^{b} .
$$

Then there exists $C>0$ such that for all $u \in \operatorname{Dom}(\mathcal{E})$ with $\|u\|_{2}=1$ and $\gamma=b+1$ one has

$$
\operatorname{Var}_{\gamma}\left(|u|^{2}\right) \mathcal{E}(u, u) \geqslant C .
$$

Proof. Let $v=\operatorname{Var}_{\gamma}\left(|u|^{2}\right)=\iint_{K \times K} d(x, y)^{\gamma}|u(x)|^{2}|u(y)|^{2} d \mu(x) d \mu(y)$, where $\gamma=$ $b+1$. There exists $y$ such that

$$
\int_{K} d^{\gamma}(x, y) u^{2}(x) d \mu(x) \leqslant v
$$


Let $r$ be defined by

$$
r=\sup \left\{s: \int_{B_{s}(y)} u^{2}(x) d \mu(x)<\frac{1}{2}\right\} .
$$

For each $s>0$ such that $\int_{B_{s}(y)}|u(x)|^{2} d \mu(x) \leqslant \frac{1}{2}$, we have

$$
v \geqslant \int_{K-B_{s}(y)} d(x, y)^{\gamma}|u(x)|^{2} d \mu(x) \geqslant s^{\gamma} \int_{K-B_{s}(y)}|u(x)|^{2} d \mu(x) \geqslant \frac{1}{2} s^{\gamma},
$$

and the definition of $r$ implies that

$$
r^{\gamma} \leqslant 2 v
$$

Moreover, by (3) there is $c>1$ (it suffices to take $c$ such that $c^{b}=9 \frac{C_{2}}{C_{1}}$ ) such that

$$
\mu\left(B_{c r}(y)\right)>8 C_{2} r^{b} .
$$

Let $t$ be an arbitrary number such that $t>r$. By the definition of $r$ we have

$$
\int_{B_{t}(y)} u^{2}(x) d \mu(x) \geqslant \frac{1}{2}
$$

which together with (3) yields

$$
\sup _{B_{t}(y)} u^{2}(x) \geq \frac{1}{2 C_{2} t^{b}} .
$$

Additionally, $\|u\|_{2}=1$ yields

$$
\inf _{B_{c t}(y)} u^{2}(x)<\frac{1}{8 C_{2} t^{b}} .
$$

Consequently, there are $x_{1}, x_{2} \in B_{c t}(y)$ such that

$$
\left(u\left(x_{1}\right)-u\left(x_{2}\right)\right)^{2} \geq \frac{1}{8 C_{2} t^{b}} .
$$

Thus, by the definition of the effective resistance metric,

$$
\mathcal{E}(u, u) \geqslant \frac{\left(u\left(x_{1}\right)-u\left(x_{2}\right)\right)^{2}}{d\left(x_{1}, x_{2}\right)} \geq \frac{1}{16 C_{2} c t t^{b}}=\frac{1}{16 C_{2} c t^{b+1}} .
$$

Since this last inequality holds for all $t>r$ we conclude that

$$
\mathcal{E}(u, u) \geqslant \frac{1}{16 C_{2} c r^{b+1}}=\frac{C_{1}^{1 / b}}{16 C_{2}^{1+1 / b} 9^{1 / b} r^{b+1}} .
$$

Now using (5) we conclude that

$$
v \mathcal{E}_{K}(u, u) \geq \frac{C_{1}^{1 / b} v}{16 C_{2}^{1+1 / b} 9^{1 / b} v^{\frac{b+1}{\gamma}}}=\frac{C_{1}^{1 / b} v}{16 C_{2}^{1+1 / b} 9^{1 / b} v}=\frac{C_{1}^{1 / b}}{16 C_{2}^{1+1 / b} 9^{1 / b}}=C,
$$

where we have used the fact $\gamma=b+1$.

Remark 1. (a) The metric measure space $(K, d, \mu)$ in Theorem 1 with the measure $\mu$ satisfying (3) is said to be an Ahlfors regular space [18. Moreover, the constant $b$ appearing in (3) is the Hausdorff dimension of $(K, d)$.

(b) Theorem 1 holds when the $K=\mathbb{R}^{1}$ with $b=1$ and is exactly the classical Heisenberg uncertainty principle of which we have given yet a different proof except for the precise value of $C$. 
(c) One can modify this proof for various cases when the properties of the space are different on small and large scales. Two such modifications are given in Theorems 2 and 3 .

Theorem 1 assumes implicitly that the space $(K, d)$ is unbounded. In particular, it is not applicable to such interesting examples as p.c.f. fractals. The next result, which is a weaker version of the previous one, generalizes the main result of 30. and deals with bounded spaces. We omit its proof since it follows from obvious modifications from the proof of Theorem 1 below.

Theorem 2. Let $K$ be a space equipped with a measure $\mu$ and the effective resistance metric $d$ associated to the Dirichlet form $\mathcal{E}$. Assume that there exist positive constants $b, C_{0}, C_{1}, C_{2}$ such that (3) holds for all $x \in K$ and all $0<r<C_{0}$.

Then there exists $C>0$ such that for all $u \in \operatorname{Dom}(\mathcal{E})$ with $\|u\|_{2}=1$ and $\gamma=b+1$ one has

$$
\operatorname{Var}_{\gamma}\left(|u|^{2}\right)(\mathcal{E}(u, u)+1) \geqslant C .
$$

Similarly, Theorem 1 excludes spaces where the local structure is significantly different from the global one, for instance, manifolds, graphs and spaces equipped with measure having atoms. In these cases the following variant of our first result can be proved using similar ideas.

Theorem 3. Let $K$ be a space equipped with a measure $\mu$ and the effective resistance metric $d$ associated to the Dirichlet form $\mathcal{E}$. Assume that there exist positive constants $b, C_{0}, C_{1}, C_{2}$ such that (3) holds for all $x \in K$ and all $r>C_{0}$. Then there exists $C>0$ such that for all $u \in \operatorname{Dom}(\mathcal{E})$ with $\|u\|_{2}=1$ one has

$$
\left(\operatorname{Var}_{\gamma}\left(|u|^{2}\right)+1\right) \mathcal{E}_{K}(u, u) \geqslant C .
$$

2.2. Weak uncertainty principle and Poincaré-type inequality. In this subsection we no longer assume that $d$ is an effective resistance metric. Instead, we will assume that a Poincaré inequality holds. No specific property of the form $\mathcal{E}$ is required for the proof; it can be taken to be an arbitrary positive-definite symmetric quadratic form on $L^{2}(K, d \mu)$. In this case the following theorem holds.

Theorem 4. Let $K$ be a space equipped with a measure $\mu$ and a metric d (not necessarily an effective resistance metric). Assume that there exists a positive constant $C_{1}$ such that the energy form $\mathcal{E}$ on $K$ satisfies the following Poincaré inequality for all locally square integrable functions $u \in \mathcal{D}$ om $(\mathcal{E})$ :

$$
\int_{B_{r}(y)}\left(u(x)-\bar{u}_{B_{r}(y)}\right)^{2} d \mu(x) \leq C_{1} r^{\gamma} \mathcal{E}(u, u),
$$

where $\bar{u}_{B_{r}(y)}$ is the average of $u$ over $B_{r}(y)$, and $\gamma$ is a positive constant. Furthermore, assume that the measure $\mu$ satisfies the "reverse volume doubling property"; that is, there exist an integer $k$ and a constant $C_{2}>1$ such that for all $x \in K$ and $r>0$ we have

$$
C_{2} \mu\left(B_{r}(x)\right) \leq \mu\left(B_{k r}(x)\right) .
$$

Then there exists $C>0$ such that for all $u \in \mathcal{D}$ omE with $\|u\|_{2}=1$ one has

$$
\operatorname{Var}_{\gamma}\left(|u|^{2}\right) \mathcal{E}(u, u) \geqslant C \text {. }
$$


Proof. The proof is similar to that of Theorem 1 with the main difference coming from the fact we need to use Poincaré's inequality instead of the basic properties of the effective resistance metric.

Let $v=\operatorname{Var}_{\gamma}\left(|u|^{2}\right)=\iint_{K \times K} d(x, y)^{\gamma}|u(x)|^{2}|u(y)|^{2} d \mu(x) d \mu(y)$, and choose $y \in$ $K$ such that $\int_{K} d(x, y)^{\gamma}|u(x)|^{2} d \mu(x) \leq v$. Define $r$ by

$$
r=\sup \left\{s: \int_{B_{s}(y)} u^{2}(x) d \mu(x)<\frac{1}{2}\right\} .
$$

Note that the definition of $r$ implies that $r^{\gamma} \leqslant 2 v$. Now, iterating (9) we can choose an integer $n>1$ and a constant $c$ such that $C_{2}^{n} \geq 16$ and $c \geq k^{n+1}$ where $C_{2}$ is the constant appearing in (9). It suffices to choose $n=\left\lfloor\frac{4 \log 2}{\log C_{2}}\right\rfloor+1$. For this choice of $c$ we have

$$
T=\frac{\mu\left(B_{c r}(y)\right)}{\mu\left(B_{k r}(y)\right)} \geq 16 .
$$

Notice that the definition of $r$ implies that $\int_{B_{k r}(y)} u^{2}(x) d \mu(x) \geq \frac{1}{2}$. Since

$$
\left|\int_{B_{c r}(y)} u(x) d \mu(x)\right| \leq \sqrt{\mu\left(B_{c r}(y)\right)}
$$

we conclude that

$$
\left|\bar{u}_{B_{c r}(y)}\right| \leq \frac{1}{\sqrt{\mu\left(B_{c r}(y)\right)}}
$$

Now, using (8) we see that

$$
\begin{aligned}
C_{1} c^{\gamma} r^{\gamma} \mathcal{E}(u, u) & \geq \int_{B_{c r}(y)}\left(u(x)-\bar{u}_{B_{c r}(y)}\right)^{2} d \mu(x) \\
& =\left\|u-\bar{u}_{B_{c r}(y)}\right\|_{L_{B_{c r}(y)}^{2}}^{2} \\
& \geq\left\|u-\bar{u}_{B_{c r}(y)}\right\|_{L_{B_{k r}(y)}^{2}}^{2} \\
& \geq\left(\|u\|_{L^{2}\left(B_{k r}(y)\right)}-\left\|\bar{u}_{B_{c r}(y)}\right\|_{L^{2}\left(B_{k r}(y)\right)}\right)^{2} \\
& =\left(\|u\|_{L^{2}\left(B_{k r}(y)\right)}-\left|\bar{u}_{B_{c r}(y)}\right| \sqrt{\mu\left(B_{k r}(y)\right)}\right)^{2} \\
& \geq\left(\frac{1}{\sqrt{2}}-\frac{1}{4}\right)^{2}=\frac{9-4 \sqrt{2}}{16} .
\end{aligned}
$$

Thus, using the fact that $r^{\gamma} \leq 2 v$ we can write

$$
v \mathcal{E}(u, u) \geq \frac{9-4 \sqrt{2}}{16} \frac{v}{C_{1} c^{\gamma} r^{\gamma}} \geq \frac{9-4 \sqrt{2}}{32} \frac{v}{C_{1} c^{\gamma} v}=\frac{9-4 \sqrt{2}}{32 C_{1} c^{\gamma}}=C .
$$

This last estimate concludes the proof.

It is interesting to observe that Theorem 4 can be proved under a slightly different form of the Poincaré inequality. In fact we have the following variant of Theorem 4 . Note that, for some applications, Theorem 5 is in a sense the strongest result of our paper, as discussed in Subsection 3.5 in relation to the analysis on groups. 
Theorem 5. The conclusion of Theorem 4 holds when condition (8) is replaced by the following modified Poincaré inequality:

$$
\int_{B_{r}(y)}\left(u(x)-u_{r}(x)\right)^{2} d \mu(x) \leq C_{1} r^{\gamma} \mathcal{E}(u, u),
$$

where $u_{r}(x)=\bar{u}_{B_{r}(x)}=\frac{1}{\mu\left(B_{r}(x)\right)} \int_{B_{r}(x)} u(y) d \mu(y)$, and $\gamma$ and $C_{1}$ are some positive constants.

Proof. We define $v, r$ and $y$ as in the proof of Theorem 4. Then, again similarly to the proof of Theorem 4, we can iterate (9) and find a constant $c>2 k$ such that

$$
\frac{\mu\left(B_{c r / 2}(y)\right)}{\mu\left(B_{k r}(y)\right)} \geq 16 \text {. }
$$

Then for any $z \in B_{k r}(y)$ we have

$$
\left|u_{c r}(z)\right| \leq \frac{1}{\sqrt{\mu\left(B_{c r}(z)\right)}} \leq \frac{1}{\sqrt{\mu\left(B_{\frac{c r}{2}}(y)\right)}} .
$$

Consequently, we have

$$
\left\|u_{c r}\right\|_{L^{2}\left(B_{k r}(y)\right)} \leq \sqrt{\frac{\mu\left(B_{k r}(y)\right)}{\mu\left(B_{c r / 2}(y)\right)}} \leq \frac{1}{4} .
$$

Now, using (11) we see that

$$
\begin{aligned}
C_{1} c^{\gamma} r^{\gamma} \mathcal{E}(u, u) & \geq \int_{B_{c r}(y)}\left(u(x)-\bar{u}_{B_{c r}(y)}\right)^{2} d \mu(x) \\
& \geq\left(\|u\|_{L^{2}\left(B_{k r}(y)\right)}-\left\|u_{c r}\right\|_{L^{2}\left(B_{k r}(y)\right)}\right)^{2} \\
& \geq\left(\frac{1}{\sqrt{2}}-\frac{1}{4}\right)^{2}=\frac{9-4 \sqrt{2}}{16} .
\end{aligned}
$$

Then the proof follows the same argument as in the proof of Theorem 4

The following proposition gives a comparison between (8) and (11) under the volume doubling condition.

Proposition 1. Assume that the measure $\mu$ satisfies the doubling volume condition; that is, there exists $C>0$ such that for all $x \in K$ and all $r>0$ we have

$$
\mu\left(B_{2 r}(x)\right) \leq C \mu\left(B_{r}(x)\right) .
$$

Let $u$ be a locally square integrable function that satisfies (8). Then u satisfies also (11).

Proof. Let $u$ be a locally square integrable function that satisfies (8). Observe that

$$
\left\|u-u_{r}\right\|_{L^{2}\left(B_{r}(y)\right)} \leq\left\|u-\bar{u}_{B_{r}(y)}\right\|_{L^{2}\left(B_{r}(y)\right)}+\left\|u_{r}-\bar{u}_{B_{r}(y)}\right\|_{L^{2}\left(B_{r}(y)\right)}=I+J .
$$

By (8) we see $I^{2} \leq C_{1} r^{\gamma} \mathcal{E}(u, u)$, where $C_{1}$ is the constant appearing in (8). To complete the proof it suffices to give a similar estimate for $J^{2}$. To simplify the 
notation, we let $V(x, r)=\mu\left(B_{r}(x)\right)$ for $x \in K$ and $r>0$. The second term in the right hand side of the last estimate can be estimated by

$$
\begin{aligned}
J^{2} & =\int_{B_{r}(y)}\left(u_{r}(x)-\bar{u}_{B_{r}(y)}\right)^{2} d \mu(x) \\
& =\int_{B_{r}(y)}\left|\frac{1}{V(x, r)} \int_{B_{r}(x)} u(t) d \mu(t)-\frac{1}{V(y, r)} \int_{B_{r}(y)} u(z) d \mu(z)\right|^{2} d x \\
& =\int_{B_{r}(y)}\left|\frac{1}{V(x, r) V(y, r)} \iint_{B_{r}(x) B_{r}(y)}(u(t)-u(z)) d \mu(z) d \mu(t)\right|^{2} d x \\
& =\int_{B_{r}(y)} M(x) d \mu(x) .
\end{aligned}
$$

For each $x \in B_{r}(y)$ we have, by Jensen's inequality,

$$
\begin{aligned}
M(x) & \leq \frac{1}{V(x, r) V(y, r)} \iint_{B_{r}(x) B_{r}(y)}|u(t)-u(z)|^{2} d \mu(z) d \mu(t) \\
& \leq \frac{1}{V(x, r) V(y, r)} \int_{B_{2 r}(x)}\left(\int_{B_{2 r}(x)}|u(z)-u(t)|^{2} d \mu(z)\right) d \mu(t) \\
& \leq \frac{4 V(x, 2 r)}{V(x, r) V(y, r)} 2^{\gamma} C_{1} r^{\gamma} \mathcal{E}(u, u)
\end{aligned}
$$

where we have used once again (8). By (12), the last inequality becomes

$$
M(x) \leq \frac{4}{V(y, r)} 2^{\gamma} C C_{1} r^{\gamma} \mathcal{E}(u, u) .
$$

Substituting this last inequality back in $J^{2}$ yields

$$
J^{2} \leq \int_{B_{r}(y)} \frac{4}{V(y, r)} 2^{\gamma} C C_{1} r^{\gamma} \mathcal{E}(u, u) d \mu(x)=4 \cdot 2^{\gamma} C C_{1} r^{\gamma} \mathcal{E}(u, u),
$$

which completes the proof of the proposition.

It is easily seen that both Theorems 4 and 5 only apply to unbounded spaces. For bounded spaces, we have the following modification of these results.

Theorem 6. Let $K$ be a space equipped with a measure $\mu$ and a metric d (not necessarily an effective resistance metric). Assume that there exist positive constants $\gamma, C_{0}, C_{1}$ and $C_{2}>1$ such that (9) together with either (8) or (11) hold for all $x \in K$ and all $0<r<C_{0}$.

Then there exists $C>0$ such that for all $u \in \mathcal{D} \operatorname{om}(\mathcal{E})$ with $\|u\|_{2}=1$ and $\gamma=b+1$ one has

$$
\operatorname{Var}_{\gamma}\left(|u|^{2}\right)(\mathcal{E}(u, u)+1) \geqslant C .
$$

Remark 2. (a) In general, and as opposed to the constant $b$ appearing in Theorem1, the constant $\gamma$ in (8) may not represent any sort of dimension of the space $(K, d)$ in the usual sense. However, in some of the examples we consider later, $\gamma$ is related to the so-called walk dimension (see [5, 9] and references therein).

(b) It is worth pointing out that in all of the results we prove in this paper, the measure $\mu$ is not necessarily a self-similar measure. But if it is self-similar, then the measure weights and the resistance scaling weights are related by a power law.

(c) Theorems 4 and 5 apply to $K=\mathbb{R}^{n}, n \geqslant 1$ with $\gamma=2$.

(d) One can show that on a metric measure space $(K, d, \mu)$ where $d$ is the effective resistance metric, and where $\mu$ satisfies (3), then the Poincaré inequality (8) holds as well as (9). Indeed, if $u$ is a function which has $L_{\mu}^{2}$-norm one in a ball of radius $r$ 
with respect to the effective resistance metric, then (3) implies that the supremum of $|u|$ has a lower bound of the form $A \cdot r^{-b / 2}$ for some positive constant $A$. If, in addition, $u$ is orthogonal to constants, then the difference between the supremum of $u$ and the infimum of $u$ also has a lower bound of the form $B \cdot r^{-b / 2}$. Then the definition of the effective resistance implies that $\mathcal{E}(u, u)$ has a lower bound of the form $C \cdot r^{-b-1}$.

(e) If $(K, d)$ is a noncompact space and $d$ is "geodesic", that is, if for any two points $x, y \in K$ there is a continuous curve $s:[0,1] \rightarrow K$ such that $s(0)=x, s(1)=y$ and $d(x, s(t))=t$, then (12) implies (91).

2.3. Weak uncertainty principle and Nash-type inequalities. In this subsection we investigate the relationship between Nash-type inequalities and the weak uncertainty principle. In particular, we first prove that the Nash inequality with small enough dimension parameters implies the weak uncertainty principle if the measure is upper $b$-regular. Furthermore, we use a Nash-type inequality to prove a weak uncertainty principle even when these dimension parameters are large. The Nash inequality plays an important role in heat kernel estimates $([8,0,16,17,22,24,36]$ and the references therein). Note that in general the Nash inequality does not imply the Poincaré inequality.

Theorem 7. Let $K$ be a space equipped with a measure $\mu$, a metric $d$ and a positivedefinite symmetric quadratic form $\mathcal{E}$ on $L_{\mu}^{2}$. Assume that the metric measure space $(K, d, \mu)$ satisfies the following upper b-regularity condition:

$$
\mu\left(B_{r}(x)\right) \leq C_{1} r^{b}, \text { for all } x \in K \text { and } r>0
$$

and the following Nash inequality:

$$
\|f\|_{2}^{2+4 / \theta} \leqslant C_{2} \mathcal{E}(f, f)\|f\|_{1}^{4 / \theta}
$$

for some positive constants $b, \theta, C_{1}$ and $C_{2}$. Furthermore assume that $\theta<2$. Then there exists $C>0$ such that for all $u \in \mathcal{D}$ omE with $\|u\|_{2}=1$ and $\gamma=\frac{2 b}{\theta}$ one has

$$
\operatorname{Var}_{\gamma}\left(|u|^{2}\right) \mathcal{E}(u, u) \geqslant C \text {. }
$$

Proof. Let $\|u\|_{2}=1$ and $v=\operatorname{Var}_{\gamma}\left(|u|^{2}\right)=\iint_{K \times K} d(x, y)^{\gamma}|u(x)|^{2}|u(y)|^{2} d \mu(x) d \mu(y)$. Then it suffices to prove that

$$
\|u\|_{1}^{4 / \theta} \leqslant C_{3} \cdot v
$$

where $C_{3}$ is a constant to be specified. By the definition of $v$, there exists $y$ such that $\int_{K} d^{\gamma}(x, y) u^{2}(x) d \mu(x) \leqslant v$. For any $r>0$ we have

$$
\|u\|_{1}=\int_{B_{r}(y)}|u(x)| d \mu(x)+\int_{K-B_{r}(y)}|u(x)| d \mu(x)=A+B .
$$


It is readily seen that $A \leq\left(\mu\left(B_{r}(y)\right)\right)^{1 / 2} \leq C_{1}^{1 / 2} r^{b / 2}$. Now we estimate $B$ as follows:

$$
\begin{aligned}
B & =\int_{K-B_{r}(y)}|u(x)| d \mu(x) \\
& =\int_{K-B_{r}(y)} d(x, y)^{-\gamma / 2} d(x, y)^{\gamma / 2}|u(x)| d \mu(x) \\
& \leq\left(\int_{K-B_{r}(y)} d(x, y)^{-\gamma} d \mu(x)\right)^{1 / 2}\left(\int_{K-B_{r}(y)} d(x, y)^{\gamma}|u(x)|^{2} d \mu(x)\right)^{1 / 2} \\
& \leq\left(\int_{K-B_{r}(y)} d(x, y)^{-\gamma} d \mu(x)\right)^{1 / 2}\left(\int_{K} d(x, y)^{\gamma}|u(x)|^{2} d \mu(x)\right)^{1 / 2} \\
& \leq v^{1 / 2}\left(\int_{K-B_{r}(y)} d(x, y)^{-\gamma} d \mu(x)\right)^{1 / 2} .
\end{aligned}
$$

The integral in the last estimate can be estimated by

$$
\begin{aligned}
\int_{K-B_{r}(y)} d(x, y)^{-\gamma} d \mu(x) & =\sum_{n \geq 0} \int_{\left\{x: 2^{n} r \leq d(x, y)<2^{n+1} r\right\}} d(x, y)^{-\gamma} d \mu(x) \\
& \leq r^{-\gamma} \sum_{n \geq 0} 2^{-n \gamma} \mu\left(B_{2^{n+1} r}(y)\right) \\
& \leq C_{1} 2^{b} r^{-\gamma+b} \sum_{n \geq 0} 2^{n(b-\gamma)} \\
& =\frac{C_{1} 2^{b}}{1-2^{b-\gamma}} r^{b-\gamma}
\end{aligned}
$$

where in the last estimate we have used the fact that $\theta<2$, which is equivalent to $b<\gamma$. Therefore,

$$
\|u\|_{1} \leqslant \frac{C_{1}^{1 / 2} 2^{b / 2}}{\sqrt{1-2^{b-\gamma}}}\left(r^{b / 2}+v^{1 / 2} r^{b / 2-\gamma / 2}\right) .
$$

The minimum of the last expression with respect to $r$ is attained when

$$
r=\left(\frac{\gamma-b}{b}\right)^{2 / \gamma} v^{1 / \gamma}
$$

which implies

or equivalently

$$
\|u\|_{1} \leqslant \frac{C_{1}^{1 / 2} 2^{b / 2}}{\sqrt{1-2^{b-\gamma}}} \frac{2 \gamma-b}{\gamma-b}\left(\frac{\gamma-b}{b}\right)^{b / \gamma} v^{\frac{b}{2 \gamma}}
$$

$$
\|u\|_{1} \leq C_{3}^{\theta / 4} v^{\theta / 4}
$$

where $C_{3}^{\theta / 4}=\frac{C_{1}^{1 / 2} 2^{b / 2}}{\sqrt{1-2^{b-\gamma}}} \frac{2 \gamma-b}{\gamma-b}\left(\frac{\gamma-b}{b}\right)^{b / \gamma}$ and $\frac{\theta}{4}=\frac{b}{2 \gamma}$.

The following variant of Theorem 7 holds for all dimension parameters.

Theorem 8. Let $K$ be a space equipped with a measure $\mu$, a metric $d$ and a symmetric quadratic form $\mathcal{E}$ on $L_{\mu}^{2}$. Assume that the metric measure space $(K, d, \mu)$ satisfies the upper b-regularity condition (13) and the following inequality:

$$
\|f\|_{2(1+2 / \theta)}^{2+4 / \theta} \leqslant C_{2} \mathcal{E}(f, f)\|f\|_{2}^{4 / \theta}
$$

for some positive constants $b, \theta, C_{1}$ and $C_{2}$. Then there exists $C>0$ such that for all $u \in \mathcal{D}$ omE with $\|u\|_{2}=1$ and $\gamma=\frac{2 b}{\theta}$ one has

$$
\operatorname{Var}_{\gamma}\left(|u|^{2}\right) \mathcal{E}(u, u) \geqslant C \text {. }
$$


Proof. Let $\|u\|_{2}=1$ and $v=\operatorname{Var}_{\gamma}\left(|u|^{2}\right)=\iint_{K \times K} d(x, y)^{\gamma}|u(x)|^{2}|u(y)|^{2} d \mu(x) d \mu(y)$. We will denote $p=1+2 / \theta$. Choose $y \in K$ such that $\int_{K} d^{\gamma}(x, y) u^{2}(x) d \mu(x) \leqslant v$.

Let $r$ be defined by

$$
r=\sup \left\{s: \int_{B_{s}(y)} u^{2}(x) d \mu(x)<\frac{1}{2}\right\} .
$$

This definition implies that $r^{\gamma} \leq 2 v$. Let $t$ be an arbitrary number such that $t>r$. Then $\int_{B_{t}(y)}|u(x)|^{2} d \mu(x) \geq \frac{1}{2}$. Consequently, we see that

$$
\frac{1}{2} \leq\left(\int_{K}|u(x)|^{2 p} d \mu(x)\right)^{1 / p}\left(\mu\left(B_{t}(y)\right)\right)^{1-1 / p},
$$

or equivalently,

$$
\frac{1}{2^{p}} \leq\|u\|_{2 p}^{2 p}\left(\mu\left(B_{t}(y)\right)\right)^{p-1}
$$

Now using the hypotheses of the theorem we obtain

$$
\begin{aligned}
\frac{1}{2^{p}} & \leq\|u\|_{2 p}^{2 p}\left(\mu\left(B_{t}(y)\right)\right)^{p-1} \\
& \leq 2 C_{2} C_{1}^{p-1} \mathcal{E}(u, u) t^{b(p-1)} \\
& =2 C_{2} C_{1}^{2 / \theta} \mathcal{E}(u, u) t^{2 b / \theta}
\end{aligned}
$$

where $C_{1}$ is the constant appearing in (13). Since this estimate holds for all $t>r$, and using the fact that $r^{\gamma} \leq 2 v$, we conclude that

$$
\frac{1}{2^{p}} \leq 2 C_{2} C_{1}^{2 / \theta} \mathcal{E}(u, u) r^{2 b / \theta} \leq 2^{1+2 b /(\theta \gamma)} C_{2} C_{1}^{2 / \theta} \mathcal{E}(u, u) v^{2 b /(\theta \gamma)}
$$

Therefore,

$$
\mathcal{E}(u, u) \operatorname{Var}_{\gamma}\left(|u|^{2}\right)^{2 b /(\theta \gamma)} \geq 2^{-p-1-2 b /(\theta \gamma)} C_{2}^{-1} C_{1}^{-2 / \theta}=C .
$$

This last inequality completes the proof by the choice of $\gamma$.

Remark 3. We wish to recall that in Theorems 7 and 8 the form $\mathcal{E}$ is not assumed to be a Dirichlet form. However, if $\mathcal{E}$ is a Dirichlet form, then it is known that (14) is equivalent to (16) for all $\theta>0$; see [1. Moreover, in this case, it follows from [36. Theorem 3.1.5] that we can estimate the volume of a ball from below, i.e., for all $x \in K$ and $r>0$ we have $\mu\left(B_{r}(x)\right) \geq C r^{\theta}$, where $C$ is a positive constant. Thus for all $r>0$ we have $C r^{\theta} \leq \mu\left(B_{r}(x)\right) \leq C^{\prime} r^{b}$, and so $\theta=b$. Consequently, if we assume in Theorem 7 that the energy form $\mathcal{E}$ is a Dirichlet form, then we can remove the restriction $\theta<2$ by using the above observation. Note that in this case, $\gamma=2$.

2.4. Local weak uncertainty principle. In this subsection we state some local versions of the results proved above. The proofs are an easy adaptation of those given above and are omitted.

Theorem 9. Let $K$ be a space equipped with a measure $\mu$, a metric $d$ and a positivedefinite symmetric quadratic form $\mathcal{E}$ on $L_{\mu}^{2}$. Assume that the metric measure space $(K, d, \mu)$ satisfies the following upper b-regularity condition (13) and the following Nash inequality:

$$
\|f\|_{2}^{2+4 / \theta} \leqslant C_{2}\left(\mathcal{E}(f, f)+\|f\|_{2}^{2}\right)\|f\|_{1}^{4 / \theta}
$$


for some positive constants $b, \theta, C_{1}$, and $C_{2}$ with $\theta<2$. Then there exists a positive constant $C>0$ such that for all $u \in \mathcal{D}$ omE with $\|u\|_{2}=1$ and $\gamma=\frac{2 b}{\theta}$ one has

$$
\operatorname{Var}_{\gamma}\left(|u|^{2}\right)(\mathcal{E}(u, u)+1) \geqslant C \text {. }
$$

Similarly, we have

Theorem 10. Let $K$ be a space equipped with a measure $\mu$, a metric $d$ and a symmetric quadratic form $\mathcal{E}$ on $L_{\mu}^{2}$. Assume that the metric measure space $(K, d, \mu)$ satisfies the upper b-regularity condition (13), and the following inequality:

$$
\|f\|_{2(1+2 / \theta)}^{2+4 / \theta} \leqslant C_{2}\left(\mathcal{E}(f, f)+\|f\|_{2}^{2}\right)\|f\|_{2}^{4 / \theta}
$$

for some positive constant $b, C_{1}$ and $C_{2}$. Then there exists $C>0$ such that for all $u \in \mathcal{D}$ om E with $\|u\|_{2}=1$ and $\gamma=\frac{2 b}{\theta}$ one has

$$
\operatorname{Var}_{\gamma}\left(|u|^{2}\right)(\mathcal{E}(u, u)+1) \geqslant C .
$$

\section{Applichtions and examples}

3.1. Sierpiński and p.c.f. fractals. As mentioned in the Introduction, the weak uncertainty principle for functions defined on the Sierpiński gasket was first introduced in [30]. While the results in that paper were stated for p.c.f. fractals, they were only proved for the Sierpiński gasket. In this subsection, we use the results of Section 2 not only to provide a simpler proof to the main results of [30, but also to establish weak uncertainty principles on all p.c.f. fractals. We briefly define the Sierpiński gasket, which is a typical example of a p.c.f. fractal, and refer to 2, 22, 39, 42, for more background on analysis on p.c.f. fractals.

Consider the contraction maps $F_{1}, F_{2}$ and $F_{3}$ defined on $\mathbb{R}^{2}$ by $F_{1}(x)=\frac{1}{2} x$, $F_{2}(x)=\frac{1}{2} x+\left(\frac{1}{2}, 0\right)$ and $F_{3}(x)=\frac{1}{2} x+\left(\frac{1}{4}, \frac{\sqrt{3}}{4}\right)$, for $x \in \mathbb{R}^{2}$. The Sierpiński gasket, $K=S G$, is the unique nonempty compact subset of $\mathbb{R}^{2}$ such that

$$
K=\bigcup_{i=1}^{3} F_{i} K
$$

For any positive integer $m, \omega=\left(\omega_{1}, \omega_{2}, \ldots, \omega_{m}\right)$ where each $\omega_{i} \in\{1,2,3\}$ is called a word of length $|\omega|=m$, and we denote $F_{\omega}=F_{\omega_{m}} \circ F_{\omega_{m-1}} \circ \ldots \circ F_{\omega_{1}}$. Then $F_{\omega} K$ is called a cell of level $m$ if $\omega$ is a word of length $m$. It is worth mentioning that $S G$ can be defined as a limit of graphs: Let $\Gamma_{0}$ be the complete graph with vertices $V_{0}=\left\{(0,0),(1,0),\left(\frac{1}{2}, \frac{\sqrt{3}}{2}\right)\right\}$, which are the fixed points of the contractions $F_{i}$. The graph $\Gamma_{m}$ with vertices $V_{m}$ is defined inductively by $V_{m}=\bigcup_{i=1}^{3} F_{i} V_{m-1}, m \geqslant 1$, and $x \sim_{m} y$ if $x$ and $y$ are in the same $m$-cell. The (standard) measure on $K$ is the probability measure on $K$ that assigns to each cell of level $m$ the measure $3^{-m}$. It follows that $S G$ is equipped with a self-similar measure that satisfies trivially (3). By defining an energy form on $S G$, it can be shown that this gives rise to a resistance metric on $S G$; see [2, 20, 22]. Consequently, Theorem 2 recovers one of the main results (Theorem 1 of 30 ) for $S G$.

More generally, let $\left\{F_{i}\right\}_{i=1}^{N}$ be a set of contractive injective maps on a compact metric space $K$ and

$$
K=\bigcup_{i=1}^{N} F_{i}(K) .
$$


We assume that $K$ is a p.c.f. self-similar set in the sense of [20, 22]. Following 20, 22], one can sometimes define a self-similar energy form $\mathcal{E}(\cdot, \cdot)$ such that

$$
\mathcal{E}(u, u)=\sum_{i=1}^{N} \rho_{i} \mathcal{E}\left(u \circ F_{i}, u \circ F_{i}\right),
$$

which gives rise to an effective resistance metric. If the resistance scaling factors $\left\{\rho_{i}\right\}_{i=1}^{N}$ satisfy the regularity condition

$$
\rho_{i}>1
$$

then the effective resistance metric induces the same topology as the original metric on $K$. Note that the energy form $\mathcal{E}(\cdot, \cdot)$ is defined without reference to any measure on $K$. If we also consider a set of positive real numbers $\left\{\mu_{i}\right\}_{i=1}^{N}$, called the measure scaling factors, such that $\sum_{i=1}^{N} \mu_{i}=1$, then we have a self-similar measure $\mu$ on $K$ such that $\mu=\sum_{i=1}^{N} \mu_{i} \mu \circ F_{i}^{-1}$. According to [22, 23, the dimension of $K$ in the effective resistance metric is the unique $b$ such that

$$
\sum_{i=1}^{N} \rho_{i}^{b}=1 .
$$

Therefore, the most natural choice of the measure scaling factors is

$$
\mu_{i}=\rho_{i}^{b} .
$$

Notice that taking $\mu_{i}=\rho_{i}^{b}$ is also natural because with this choice the asymptotic behavior of the Weyl function is well studied [19, 22. Our Theorem 2 holds on $K$ if the condition (3) is satisfied. The difficulty is, however, that if the measure scaling factors are not equal and some combinatorial-geometric conditions are not satisfied, then in many cases the self-similar measure $\mu$ does not satisfy even the volume doubling property much less the regularity condition (3). Fortunately, for many cases the regularity condition (3) does hold, such as in the situation of the nested fractals. Many related questions, and the Nash inequality in this context in particular, are discussed in [24.

To construct noncompact fractals which satisfy the conditions of Theorem 1, we assume for simplicity that $\left\{F_{i}\right\}_{i=1}^{N}$ are contractive injective maps on $\mathbb{R}^{d}$ and $K$ is the unique compact set such that $K=\bigcup_{i=1}^{N} F_{i}(K)$. Then one can construct an increasing sequence of sets $K_{n}$ using inverse maps $F_{i_{n}}^{-1}$, and define the blowup of $K$ to be $K_{\infty}=\bigcup_{n=0}^{\infty} K_{n}$ where $K_{0}=K$. Then $K_{\infty}$ is an unbounded self-similar set, called fractal blowup, and was first introduced in [38; see also [35, 34, 33, 43. for more about fractal blowups.

3.2. Sierpiński graphs. As another application of the results of Section 2, we prove a weak uncertainty principle of some graphs related to the Sierpiński gasket $K=S G$, and its blowup $K_{\infty}$. More precisely, for any integer $m \geqslant 0$, let $\Gamma_{m}$ be the $m t h$ pre-gasket approximation to $K$, i.e., the $m t h$ graph approximation of $K$. We define a (finite) graph $\Gamma_{-m}$ by $\Gamma_{-m}=F_{\omega_{i}}^{-1} \circ F_{\omega_{2}}^{-1} \circ \ldots \circ F_{\omega_{m}}^{-1}\left(\Gamma_{m}\right)$, and an infinite graph $\Gamma_{\infty}$ by $\Gamma_{\infty}=\bigcup_{m \geqslant 0} \Gamma_{-m}$. The graph $\Gamma_{\infty}$ is an example of an infinite self-similar graph, which is also referred to as the Sierpiński lattice; we refer to [43] and the references therein for more on this type of graph. Note that for all integers $m \geqslant 0, \Gamma_{-m}$ is similar to the (finite) graph obtained by taking $F_{\omega_{i}}=F_{1}$ for all $i$, 
in which case, $\Gamma_{-m}=2^{m} \Gamma_{m}$. In this case it can be shown that Theorem 3 holds on $\Gamma_{\infty}$.

3.3. Uniform finitely ramified fractals and graphs. The uniform finitely ramified fractals (u.f.r.) and the unbounded fractals associated with them were introduced in [17] and include the nested fractals and are contained in the class of p.c.f. self-similar sets; see [17, 22, 20]. Clearly, Theorems 1 and 2 apply to these class of fractals.

Additionally, the (infinite) u.f.r. graphs were constructed from u.f.r. in [17, where it was proved that there exists an effective resistance metric on this class of graphs. Therefore, using [17, Lemma 3.2] one can show that Theorem 3 applies in this setting as well.

3.4. Sierpiński carpets and graphical Sierpiński carpets. These are examples of non-finitely-ramified fractals and fractal graphs [3, 4, 5, 6, 26. In particular, they are non-p.c.f. fractals, and it is interesting to notice that most of our results apply in this setting. Hence, we answer affirmatively a question posed in 30] of whether the main results of that paper apply to "genuine" non-p.c.f. fractals. More precisely, on the generalized Sierpiński carpets (GSC) and the unbounded sets that can be constructed based on them, it is known that a two sided heat kernel estimate holds, [3, 5, 7. Thus, following [3, 5, 7, 9] or [15, Theorem 3.2], one can show that (3) holds on the GSC and all related sets; this in turn implies that (9) holds also in these settings. Moreover, it is known that the two sided heat kernel estimate implies that the Poincaré inequality holds; e.g., see [6, 9]. Consequently, Theorem 4 applies to all unbounded spaces constructed on GSC, while Theorem 6 applies to all Sierpiński carpets for which such estimates exist. Moreover, Theorem 1 applies to resistance Dirichlet forms on the Sierpiński carpets in dimension less than 2, such as self-similar Dirichlet forms on the Sierpiński carpets constructed in [26].

3.5. Groups. Let us briefly describe how our results apply in the case when the underlying space $K=G$ is a group and the distance $d$ and quadratic form $\mathcal{E}$ have some invariance properties.

For instance, let $G$ be a real connected Lie group equipped with a left-invariant Riemannian structure (in fact, a left-invariant sub-Riemannian structure would work as well); see, e.g., 36, 44 for details. We let $d$ be the Riemannian distance and $\mathcal{E}(f, f)=\int_{G}|\nabla f|^{2} d \mu$ where $\mu$ is the (left-invariant) Riemannian measure and $|\nabla f|^{2}$ the Riemannian length of the gradient of $f$. In this setting, Theorem 1 and Theorem 7 apply only to the case $G=\mathbb{R}$ because in higher dimensions the resistance metric is infinite. Observe that the reverse doubling condition (9) holds on any noncompact Lie group. Therefore, Theorem 4 applies with $\gamma=2$ to all the cases where $G$ is noncompact with polynomial volume growth. This includes $\mathbb{R}^{n}$ and all nilpotent Lie groups, in particular the Heisenberg group

$$
\left\{\left(\begin{array}{ccc}
1 & x & z \\
0 & 1 & y \\
0 & 0 & 0
\end{array}\right): x, y, z \in \mathbb{R}\right\} .
$$

We do not know if condition (8) implies polynomial growth, but it seems unlikely that it holds for groups of exponential growth. 
Theorem 5 has the great advantage over Theorem 4 that it applies to all noncompact unimodular Lie groups. This is because (11) holds with $\gamma=2$ on any such Lie group; see, e.g., [36, Theorem 3.3.6].

Finally, because of Remark 3, Theorem 8 is essentially restricted to the case when $G=\mathbb{R}^{n}$ for some $n=\theta$.

Consequently, in the case of Lie groups, Theorem 5 is by far the most powerful result. Moreover, Theorem[5]applies as well to the case of infinite, finitely generated groups equipped with the counting measure and a quadratic form of the type

$$
\mathcal{E}(f, f)=\frac{1}{2|S|} \sum_{x \in G} \sum_{s \in S}|f(x s)-f(x)|^{2}
$$

where $S=S^{-1}$ is a finitely symmetric generating set. Indeed, in this setting, (11) holds with $\gamma=2$ (the proof of [36, Theorem 3.3.6] may easily be adapted to this setting).

3.6. Metric measure spaces and heat kernel estimates. Our results of Section 2 are applicable to the general setting of metric measure spaces. For a metric measure space $(K, d, \mu)$ the main assumption we make is the existence of a heat kernel $\left\{p_{t}\right\}_{t>0}$, which is the fundamental solution of the heat equation where the selfadjoint operator associated with the energy form $\mathcal{E}$ plays the role of a Laplacian. If the heat kernel, which is a nonnegative measurable function $p_{t}(x, y)$ on $[0, \infty) \times K \times K$, satisfies the following two sided estimate for $\mu$-almost $x, y \in K$ and all $t \in(0, \infty)$ :

$$
\frac{1}{t^{\alpha / \beta}} \Phi_{1}\left(\frac{d(x, y)}{t^{1 / \beta}}\right) \leqslant p_{t}(x, y) \leqslant \frac{1}{t^{\alpha / \beta}} \Phi_{2}\left(\frac{d(x, y)}{t^{1 / \beta}}\right),
$$

where $\alpha$ is the Hausdorff dimension of $(K, d)$ and $\beta=\alpha+1$, and $\Phi_{1}, \Phi_{2}$ are nonnegative monotone decreasing functions on $[0, \infty)$, then under a mild decay condition on $\Phi_{2}$, it is shown in [15. Theorem 3.2] that (20) implies (3) with $b=\alpha$. This can be used in turn to prove (9). Consequently, once a Poincaré-type estimate is established in this setting, our results can be applied.

Moreover, heat kernel estimates of the type

$$
\begin{aligned}
\frac{c_{1}}{\mu\left(B\left(x, t^{1 / \gamma}\right)\right)} \exp \left(-\left(\frac{d(x, y)^{\gamma}}{c_{1} t}\right)^{\frac{1}{\gamma-1}}\right) & \leqslant p_{t}(x, y) \\
\leqslant & \leqslant \frac{c_{2}}{\mu\left(B\left(x, t^{1 / \gamma}\right)\right)} \exp \left(-\left(\frac{d(x, y)^{\gamma}}{c_{2} t}\right)^{\frac{1}{\gamma-1}}\right)
\end{aligned}
$$

imply the Poincaré inequality, and these estimates can be established on many fractals and other spaces (see [9] and the references therein).

\section{ACKNowledgments}

The authors are grateful to Martin Barlow, Richard Bass and Robert Strichartz for many helpful discussions, and to the anonymous referee(s) for suggesting to consider the Nash inequality in this context and noticing the connection of our work with preprint [1]. 


\section{REFERENCES}

[1] D. Bakry, T. Coulhon, M. Ledoux, and L. Saloff-Coste, Sobolev inequalities in disguise, Indiana Univ. Math. J. 44 (1995), no. 4, 1033-1073. MR1386760 (97c:46039)

[2] M. T. Barlow, Diffusion on Fractals, in: Lecture Notes in Mathematics, Vol. 1690, Springer, Berlin, 1998. MR.1668115 (2000a:60148)

[3] M. T. Barlow and R. F. Bass, The construction of Brownian motion on the Sierpiński carpet. Ann. Inst. H. Poincaré Probab. Statist. 25 (1989), 225-257. MR1023950 (91d:60183)

[4] M. T. Barlow and R. F. Bass, On the resistance of the Sierpiński carpet. Proc. Roy. Soc. London Ser. A 431 (1990), 345-360. MR.1080496 (91h:28008)

[5] M. T. Barlow and R. F. Bass, Brownian motion and harmonic analysis on Sierpinski carpets. Canad. J. Math., 51 (1999), 673-744. MR1701339(2000i:60083)

[6] M. T. Barlow and R. F. Bass, Random walks on graphical Sierpinski carpets,. Random walks and discrete potential theory (Cortona, 1997), 26-55, Sympos. Math., XXXIX, Cambridge Univ. Press, Cambridge, 1999. MR 1802425 (2002c:60116)

[7] M. T. Barlow and R. F. Bass, Transition densities for Brownian motion of the Sierpinski carpet, Probab. Theory Relat. Fields, 91 (1992), 307-330. MR.1151799 (93k:60203)

[8] M. T. Barlow and R. F. Bass, Stability of parabolic Harnack inequalities. Trans. Amer. Math. Soc., 356 (2004), 1501-1533. MR2034316 (2005e:60167)

[9] M. T. Barlow, R. F. Bass and T. Kumagai, Stability of parabolic Harnack inequalities on metric measure spaces. J. Math. Soc. Japan, 58 (2006), 485-519. MR2228569 (2007f:60064)

[10] M. T. Barlow and J. Kigami, Localized eigenfunctions of the Laplacian on p.c.f. self-similar sets, J. London Math. Soc., 56 (1997), no. 2, 320-332. MR1489140 (99b:35162)

[11] P. Ciatti, F. Ricci, and M. Sundari, Heisenberg-Pauli-Weyl Uncertainty Inequalities and Polynomial Volume Growth, to appear in Advances in Mathematics.

[12] M. Fukushima and T. Shima, On a spectral analysis for the Sierpinski gasket, Potential Anal., 1 (1992), 1-35. MR.1245223 (95b:31009)

[13] G. B. Folland and A. Sitaram, The uncertainty principle: A mathematical survey, J. Fourier Anal. Appl., 3 (1997), no. 3, 207-238. MR1448337 (98f:42006)

[14] A. Grigoŕyan and A. Telcs, Sub-Gaussian estimates of heat kernels on infinite graphs, Duke Math. J., 109 (2001), no. 3, 451-510. MR1853353 (2003a:35085)

[15] A. Grigoŕyan, J. Hu and K. Lau, Heat kernels on metric measure spaces and an application to semilinear elliptic equations, Trans. Amer. Math. Soc., 355 (2003), no. 5, 2065-2095. MR:1953538 (2003j:60103)

[16] A. Grigoŕyan, Heat kernel upper bounds on fractal spaces, preprint.

[17] B. M. Hambly and T. Kumagai, Heat kernel estimates for symmetric random walks on a class of fractal graphs and stability under rough isometries, Proc. Symp. Pure Math., 72 (2004), 233-259. MR2112125 (2005k:60141)

[18] J. Heinonen, "Lectures on analysis on metric spaces," Universitext, Springer-Verlag, New York, 2001. MR 1800917 (2002c:30028)

[19] J. Kigami and M. L. Lapidus, Weyl's problem for the spectral distribution of Laplacians on p.c.f. self-similar fractals. Comm. Math. Phys. 158 (1993), 93-125. MR1243717(94m:58225)

[20] J. Kigami, Harmonic calculus on p.c.f. self-similar sets. Trans. Amer. Math. Soc. 335 (1993), 721-755. MR1076617 (93d:39008)

[21] J. Kigami, Effective resistances for harmonic structures on p.c.f. self-similar sets. Math. Proc. Cambridge Philos. Soc. 115 (1994), 291-303. MR1277061 (95h:28012)

[22] J. Kigami, "Analysis on Fractals," Cambridge University Press, New York, 2001. MR1840042 (2002c:28015)

[23] J. Kigami, Harmonic analysis for $r$ esistance forms. J. Functional Analysis 204 (2003), 399-444. MR2017320 (2004m:31010)

[24] J. Kigami, Local Nash inequality and inhomogeneity of heat kernels, Proc. London Math. Soc. (3) 89 (2004), 525-544. MR2078700 (2005f:47105)

[25] B. Krön and E. Teufl, Asymptotics of the transition probabilities of the simple random walk on self-similar graphs, Trans. Amer. Math. Soc., 356 (2004), no. 1, 393-414. MR.2020038 (2004k:60130)

[26] S. Kusuoka and X. Y. Zhou, Dirichlet forms on fractals: Poincaré constant and resistance. Probab. Theory Related Fields 93 (1992), 169-196. MR.1176724 (94e:60069) 
[27] S. Kusuoka and X. Zhou, Waves on fractal-like manifolds and effective energy propagation. Probab. Theory Related Fields 110 (1998), 473-495. MR1626955 (99i:58148)

[28] L. Malozemov and A. Teplyaev, Pure point spectrum of the Laplacians on fractal graphs. J. Funct. Anal. 129 (1995), 390-405. MR.1327184 (96e:60114)

[29] L. Malozemov and A. Teplyaev, Self-similarity, operators and dynamics. Math. Phys. Anal. Geom. 6 (2003), 201-218. MR1997913 (2004d:47012)

[30] K. A. Okoudjou and R. S. Strichartz, Weak uncertainty principles on fractals, J. Fourier Anal. Appl., 11 (2005), no. 3, 315-331. MR2167172 (2006f:28011)

[31] J. F. Price and P. C. Racki, Local uncertainty inequalities for Fourier series, Proc. Amer. Math. Soc., 93 (1985), 245-251. MR770530(86e:42018)

[32] C. Sabot, Electrical networks, symplectic reductions, and application to the renormalization map of self-similar lattices. Fractal geometry and applications: A jubilee of Benoît Mandelbrot. Part 1, 155-205, Proc. Sympos. Pure Math. 72, Amer. Math. Soc., 2004. MR2112106 (2005m:34202)

[33] C. Sabot, Laplace operators on fractal lattices with random blow-ups. Potential Anal. 20 (2004) 177-193. MR2032947 (2004m:47082)

[34] C. Sabot, Spectral properties of self-similar lattices and iteration of rational maps. Mém. Soc. Math. Fr. (N.S.) No. 92 (2003), vi+104 pp. MR1976877 (2004d:82016)

[35] C. Sabot, Pure point spectrum for the Laplacian on unbounded nested fractals. J. Funct. Anal. 173 (2000) 497-524. MR1760624 (2001j:35216)

[36] L. Saloff-Coste, "Aspects of Sobolev-type inequalities," London Mathematical Society Lecture Note Series, 289. Cambridge University Press, 2002. MR.1872526 (2003c:46048)

[37] R. S. Strichartz, Uncertainty principles in harmonic analysis, J. Funct. Anal., 84 (1989), 97-114. MR.999490 (91a:42017)

[38] R. S. Strichartz, Fractals in the large, Canad. J. Math., 50 (1998), no. 3, 638-657. MR1629847 (99f:28015)

[39] R. S. Strichartz, Analysis on fractals, Notices Amer. Math. Soc., 46 (1999), 1199-1208. MR.1715511 (2000i:58035)

[40] R. S. Strichartz, Function spaces on fractals, J. Funct. Anal., 198 (2003) 43-83. MR.1962353 (2003m:46058)

[41] R. S. Strichartz, Fractafolds based on the Sierpiński gasket and their spectra, Trans. Amer. Math. Soc. 355 (2003), 4019-4043. MR.1990573 (2004b:28013)

[42] R. S. Strichartz, Fractal Differential Equations: A Tutorial, Princeton University Press, 2006. MR.2246975 (2007f:35003)

[43] A. Teplyaev, Spectral Analysis on Infinite Sierpiński Gaskets, J. Funct. Anal., 159 (1998), 537-567. MR1658094 (99j:35153)

[44] N. Th. Varopoulos, L. Saloff-Coste, and T. Coulhon, "Analysis and geometry on groups," Cambridge Tracts in Mathematics, 100, Cambridge University Press, Cambridge, 1992. MR:1218884 (95f:43008)

Department of Mathematics, University of Maryland, College Park, Maryland $20742-4015$

E-mail address: kasso@math.umd.edu

Department of Mathematics, Malott Hall, Cornell University, Ithaca, New York $14853-4201$

E-mail address: lsc@math.cornell.edu

Department of Mathematics, University of Connecticut, Storrs, Connecticut 062693009

E-mail address: teplyaev@math.uconn.edu 